\title{
Transplantation Research and Medicine
}

REVIEW ARTICLE

\section{The Bone in Renal Transplant Recipients-A Nephrological and Orthopedic Review}

\author{
Natacha Rodrigues $^{1 * \#}$, Filipe Rodrigues ${ }^{2 \#}$, Alice Santana $^{1}$ and Pedro Neves ${ }^{2}$ \\ ${ }^{1}$ Nephrology and Renal Transplantation Department, Centro Hospitalar Universitário Lisboa Norte, Portugal \\ ${ }^{2}$ Orthopedics Department, Centro Hospitalar Universitário do Porto, Portugal \\ ${ }^{\#}$ Equal Contribution.
}

*Corresponding author: Natacha Rodrigues, MD., Nephrology and Renal Transplantation Department, Centro Hospitalar Universitário Lisboa Norte, Portugal, Tel: 00351-917279060

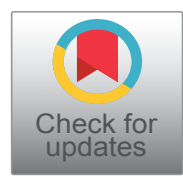

\begin{abstract}
The definition of Chronic Kidney Disease - Mineral Bone Disorder (CKD-MBD) has suffered significant changes over the last decade as our knowledge on the matter grows. The complexity of this subject is even bigger if you consider renal transplant recipients (RTRs) - they already have a legacy of CKD-MBD previous to transplant, their bones will be under the direct and indirect effects of immunosuppression and they will develop CKD-MBD secondary to their graft (dis) function. All these issues occur in addition to the risk factors of the general population for bone disease. Diagnosis and management of CKD-MBD in RTRs is difficult, several tools are yet to be validated and bone biopsy is not widely available. In the past years, new therapeutic strategies have been proposed for these patients. Special consideration should be given to the orthopedic management in RTRs regarding fracture risk and avascular necrosis of the major joints, particularly the femoral head, due to the effects of immunosuppression.
\end{abstract}

\section{Keywords}

Transplantation, CKD-MBD, Bone biopsy, Immunosuppression, Avascular necrosis, osteoarthritis, Bisphosphonates, Denosumab, Orthopedics

\section{CKD-MBD in CKD Patients before Renal Trans- plantation}

\section{Definition}

The Kidney Disease Improving Global Outcome (KDI$\mathrm{GO}$ ) has greatly contributed to the CKD-MBD's definition in CKD population. Since 2009 it is consensual that CKD-MBD includes three distinct aspects [1]: Laborato- ry abnormalities - changes in calcium, phosphorus, PTH and vitamin D metabolism. Bone abnormalities - changes in bone turnover, mineralization and volume graded accordingly to TMV classification, with bone biopsy playing a major role. Vascular calcifications and soft tissue calcifications - with classification systems according to radiologic aspects.

The need to include all three aspects arose from the evidence that all of them, combined or individually, led to a significant increase of the risk for cardiovascular disease, fractures and mortality [1]. In 2017 the definition was updated reinforcing these three components of CKD and the associations mentioned above [2].

\section{Physiopathology}

As it is now assumed, the main event for CKD-MBD in CKD population is the reduced phosphate excretion as glomerular filtration rate reduces. The chronic phosphate retention stimulates the production of two important phosphaturic hormones: FGF-23 and PTH [3].

FGF-23 acts on phosphate levels interfering with both absorption and excretion [4]. On one hand it inhibits the activation of 25-dihydroxy vitamin $D$ into 1,25-dihydroxy vitamin $D$ (calcitriol) - by inhibiting 1-alfa hydroxylase and stimulating its catabolizing enzyme 24,25-hydroxylase - and so, inhibits the absorption of phosphorus and calcium in the gut by reducing sodium-phosphate co-transporter $\mathrm{NaPi}_{2} \mathrm{~b}$ in the gut. Reduced levels of 1,25-dihydroxy vitamin $D$ are also a

Citation: Rodrigues N, Rodrigues F, Santana A, Neves P (2019) The Bone in Renal Transplant Recipients-A Nephrological and Orthopedic Review. Int J Transplant Res Med 5:045. doi.org/10.23937/25724045.1510045 
stimulus for PTH secretion [5]. On the other hand, potentialized by Klotho, FGF-23 stimulates phosphate excretion in the kidney by mobilizing sodium-phosphate co-transporters that control phosphate transport in proximal tubular epithelial cells ( $\mathrm{NaPi} 2 \mathrm{a}$ and $\mathrm{NaPi} 2 \mathrm{c}$ ) $[6,7]$.

Stimulated by chronic phosphate excretion and reduced serum levels of calcium secondary to reduced 1,25-dihydroxy vitamin $D$, parathyroid gland's chief cells will produce and secret PTH [8-10]. PTH acts on bone, kidney and intestine. PTH binds to osteoblasts stimulating their expression of RANK Ligand [11]. RANK Ligand will bind to osteoclast precursors ' RANK and stimulate their fusion, forming new osteoclasts, which ultimately enhances bone resorption. In the kidney, PTH promotes calcium reabsorption in the distal tubules and renal collecting tubules, inhibits phosphate tubular reabsorption by inhibiting sodium-phosphate co-transporters. Also, PTH stimulates the conversion of 25-hydroxy vitamin D into 1,25-dihydroxy vitamin D which will stimulate calcium uptake from the intestine [12].

Due to all these mechanisms, most patients will have high serum levels of FGF23 and PTH and low serum levels of 1,25-dihydroxy vitamin $D$ by CKD stage 3 [13]. As glomerular filtration rate diminishes, these mechanisms are not capable of maintaining homeostasis causing serum phosphate to rise and serum calcium to diminish by stage 4 [13].

The bone metabolic impact of these hormones and ions, together with the retention of pro-inflammatory molecules prone to calcification processes, lead to bone abnormalities - changes in bone turnover, mineralization and volume - vascular calcifications and soft tissue calcifications.

It has been well established that CKD stimulates vascular calcification - both intimal and medial. Intimal calcification in CKD is observed through atherosclerotic plaque neointimal calcification produced by osteoblastic transition of cells in the neointima whose origin have been linked to smooth muscle cells and circulating mesenchymal cells $[14,15]$. Medial calcification in CKD has been associated to vascular smooth muscle cells undergoing chondro-osseous transition $[16,17]$.

Many molecules such as DKK1, sclerostin, bone morphogenetic protein- 9 and active in have been linked in preclinical studies to CKD stimulated vascular calcification and vascular osteoblastic transition. Better characterization of these interactions with clinical studies wait for their results [18-20].

\section{CKD-MBD in CKD Patients after Renal Trans- plantation}

\section{Mineral and hormonal modifications}

When a functioning kidney is implanted in a patient with CKD stage 5, high levels of FGF23 and PTH will suddenly act on a responsive organ, resulting in an important and immediate decrease of phosphate levels, immediate rise of calcium levels, progressive rise in 1,25-dihydroxy vitamin $D$ and, by feedback, a theoretically normalization of FGF 23 and PTH [21,22].

According to several studies, hypophosphatemia is registered in more that $90 \%$ of RTRs in the first month. This is the result of the inhibition of phosphate reabsorption in proximal tubule by FGF 23, PTH and probably other phosphatonines $[23,24]$. It has been demonstrated a stronger correlation between hypophosphatemia and FGF 23 levels in the first month, and a stronger correlation with PTH levels the following months.

Hypercalcemia is observed more often between the third and fifth month after kidney transplantation (30$50 \%$ of patients) [25]. It is related to a progressive rise of 1,25 dihydroxy vitamin $D$ and can also be sustained in patients with previous parathyroid hyperplasia. High calcium levels are normally asymptomatic, rarely severe, with only anecdotic cases of acute allograft dysfunction or calciphylaxis related to hypercalcemia.

FGF-23 levels tend to follow an initial abrupt decrease beginning almost immediately after transplantation and lasting the first three months (reduction of around $90 \%$ of the initial level by the third month [24]. After this period the decrease in FGF-23 serum levels tends to be much slower. The correlation between hypophosphatemia and FGF-23 levels is particularly well established on the first month after transplantation, suggesting that it can be the most contributive factor for hypophosphatemia during this period [24].

Over the first six months after renal transplantation PTH levels decrease almost half of its pre-transplant level. After the first year, at least one third of patients still have high levels of PTH [26]. This is mainly explained by hyperplasia of parathyroid gland previous to transplantation with less calcium sensing receptors in the gland, resulting a decreased response to hypercalcemia. In less frequent cases, tertiary hyperparathyroidism installed previously to transplant can lead to severe bone disease, with the development of brown tumors only manageable with parathyroidectomy in ever rarer situations [27].

Some studies have shown a clinical association between high levels of PTH and/or FGF-23 with clinical outcomes. An analysis of 1609 RTRs showed that persistent hyperparathyroidism was independently associated with worse graft survival [28]. Hyperparathyroidism also correlates with significant bone loss at the hip [29]. A single center study with 140 patients showed that persistent hyperparathyroidism on the third month after transplant was an independent risk factor for fracture with a 7.5 fold increase risk [30]. Also, a study involving 177 RTRs compared 
osteodensitometry at day 14 and day 264 post-renal transplantation and concluded that higher levels of FGF-23 at the time of the transplant were associated with higher risk for bone mineral density loss during the first-year post-transplant [31].

\section{Glomerular filtration rate after renal transplant}

Unfortunately, not all RTRs have a completely functional graft. After transplant, patients can have different glomerular filtration rates according to several factors related to the donor, the receptor and the surgery itself. Also, with time and eventual post-transplant complications, glomerular filtration rate can decrease leading to a reduction in phosphate excretion. When this happens, patients will start to activate the same hormonal and feedback mechanisms as non-transplant CKD patients with the same glomerular filtration rates. This is a very important issue because doctors tend to focus on the particularities of RTRs (immunosuppression, infections, neoplasms) and often forget to approach anemia, hypertension, CKD-MBD common to non-transplant CKD patients.

\section{Immunosuppression}

Corticotherapy is responsible not only for the inhibition of osteoblast proliferation and differentiation but also for the stimulation of apoptosis of either osteoblasts or osteocytes. Whilst hyperparathyroidism tends to affect predominantly cortical bone, corticosteroids tend to affect predominantly trabecular bone of the axial skeleton $[32,33]$. Even small dosages of prednisolone (as low as 5 to $7.5 \mathrm{mg} / \mathrm{d}$ ) have been associated with significant reduction of bone density and increase in fracture rate [34]. Although osteoporosis has been related to the cumulative dose exposure in kidney transplant recipients [33], steroid-free regimen has a greater incidence of rejection episodes, hence the application of high dose corticotherapy.

Calcineurin inhibitors can be responsible for Calcineurin inhibitor-induced pain syndrome (CIPS), a rare adverse effect that greatly impairs the patient's functions and abilities. CIPS symptoms include deep pain, usually symmetrical in the lower extremities, and are associated with high levels of CNI [35]. Although there is experimental evidence of calcineurin inhibitors' adverse effects on osteoblasts and osteoclasts [36,37], more scientific evidence is lacking.

Recent experimental studies have shown that inhibition of mTORC1 signaling by rapamycin impaired both proliferation and osteogenic differentiation of mouse bone marrow stromal cells in vitro, causing trabecular bone loss in vivo [38,39]. The implications of these findings in RTRs are yet to be clinically established.

\section{Risk factors in the general population}

Bone disease in RTRs has not only the contribution of the above CKD and transplant specific factors, but also the contribution of risk factors common to the general population.

The most prevalent bone disease worldwide is osteoporosis, characterized by low bone mass (BMD), deterioration of bone tissue, and disruption of bone microarchitecture leading to compromised bone strength and an increased fracture risk [40]. Accordingly to the World Health Organization (WHO) criteria, osteoporosis is defined as a BMD that lies 2.5 standard deviations or more below the average value for young healthy women (a T-score of <-2.5 SD) [41,42]. Osteoporosis affects an enormous number of people (of both sexes and all races), and its prevalence will increase as the population ages.

There are some factors that increase fracture risk and worsen osteoporosis, independent (ly) of previous bone mineral density [43]. There is a well established association with age [44] and low body mass index $\left(\mathrm{BMI}<21 \mathrm{~kg} / \mathrm{m}^{2}\right)$ and post-menopausal status [45]. A history of a previous osteoporotic fracture is another important factor for further fracture risk and almost doubles the risk of spinal fractures [46], as well as parental history of hip fracture [47], smoking [48] and oral glucocorticoids $\geq 5 \mathrm{mg} / \mathrm{d}$ of prednisone for $>3$ months (ever) $[49,50]$. Also, there is a dose-dependent relationship between alcohol intake and fracture risk. Daily intake of 3 or more units of alcohol is associated with an increased fracture risk [51].

\section{Monitoring and Diagnosis}

KDIGO guidelines recommend that, in the immediate post-kidney-transplant period, serum calcium and phosphorus should be measured and monitored atleast weekly, until stable levels are reached [1]. Hypophosphatemia should only be corrected with supplements only if patients are symptomatic or have very low levels because supplements can rise the already high PTH levels resulting in an important hypercalcemia as a response to a functional kidney.

In the first three months after kidney transplant, calcium and phosphate levels should be measured weekly and PTH and vitamin D should be measured monthly [1].

After the immediate post-kidney-transplant period, it is reasonable to establish the frequency of serum calcium, phosphorus, and PTH measurements based on the magnitude of abnormalities, as well as the rate of progression of CKD.

As established to the CKD population in general:

- Patients with CKD stage 1-3T should have their calcium and phosphorus levels measured every 6-12 months and PTH according to basal level and progression of CKD [2]. 
- Patients with CKD stage 4T should have their calcium and phosphorus levels measured every 3-6 months and PTH every 6-12 months [2].

- Patients with CKD stage 5T should have their calcium and phosphate levels measured every 1-3 months and PTH levels every 3-6 months [2].

- Alkaline phosphatase should be measured in patients with CKD stage 3-5T annually, or more frequently if the patient has hyperparathyroidism [2].

The most widely validated technique to measure $B M D$ in the general population is dual energy $X$-ray absorptiometry (DXA). Osteoporosis diagnostic criteria are based on the T-score for BMD measured by DXA as well as the recommended entry criterion for the development of pharmaceutical interventions $[52,53]$. In the last decade it has been discussed the role of DXA in RTRs. It has been advocated that this exam should be done in the first three months to first year in RTRs, especially if they receive corticosteroids or have risk factors for osteoporosis [1,2]. Still, the predictive risk factor for fracture in this population using this exam is not yet validated.

In the general population, some tools are strongly validated to estimate a ten-year probability of major osteoporotic fractures without the need of DXA - like the Fracture Risk Assessment Tool (FRAX). Still, it is not yet validated for RTRs patients.

The gold standard to diagnose bone disease in general CKD patients (including RTRs) is bone biopsy with double tetracycline labeling [54-56]. This exam continues to be very difficult to perform routinely because of its logistics - being an invasive procedure, patients tend to refuse, and also the processing and analysis of bone biopsies require considerable expertise [57], which confines the exam only to reference centers. Still, because of the high number of case reports in RTRs evidencing the lack of correlation between PTH and bone biopsy diagnosis, it has been strongly advised in some situations as persistent bone pain, fragility fractures or severe osteoporosis to exclude adynamic bone disease before initiation of antiresorptive therapy $[1,2]$.

\section{Nephrological Management}

Lifestyle advice is consensual and applicable to all patients. Professionals should give advice concerning smoking cessation, alcohol intake reduction, mobilization increase and fall prevention. It should also be considered corticosteroid minimization in high risk patients (when possible).

Concerning the bone, KDIGO guidelines $[1,2]$ recommend DXA in RTRs at three months after renal transplant if glomerular filtration rate $>30 \mathrm{ml} / \mathrm{min} / 1.73 \mathrm{~m}^{2}$. Those with low BMD (T-score $<2.5)$ should have a bone biopsy to exclude adynamic bone disease before starting anti-resorptive therapy. Those with intermediate to normal BMD (T-score $>2.5$ ) should receive vitamin D supplementation and calcium and repeat DXA after 2 years. RTRs with glomerular filtration rate $<30 \mathrm{ml} /$ $\mathrm{min} / 1.73 \mathrm{~m}^{2}$ should be managed as CKD 4-5 not on dialysis.

Concerning biochemical disorders, focus is made on calcium and phosphate. Treating hypercalcemia should have in consideration PTH levels. If PTH levels are low or normal, a conservative strategy should be adopted because it tends to resolve in the first three months with no need for specific therapy. If PTH levels are high, hypercalcemia can be considered a direct consequence of PTH action, taking in consideration the use of calcimimetics or even parathyroidectomy in refractory secondary hyperparathyroidism.

It is important to emphasize that the above recommendations are classified as level 2 evidence.

Measurement and correction of vitamin D serum levels are suggested in the literature despite its low grade of evidence. This monitorization should be made based on the baseline values and interventions, targeting not only the renal impact but also the pleiotropic effects of this hormone in the general population - like its known relation with cardiovascular disease, auto-immune diseases, neoplasms and insulin resistance.

Calcimimetic cinacalcet was found to reduce PTH significantly in RTRs but there was no evidence of improving graft function [58] or having a beneficial impact on bone mineralization in this population [59].

Antiresorptive agents induce low bone turnover. Bisphosphonates accumulate at sites of active bone resorption. In addition to their ability to inhibit calcification, bisphosphonates inhibit hydroxyapatite breakdown, thereby effectively suppressing bone resorption [60]. Renal excretion compromises drug half-life and pharmacokinetics in CKD, however treatment seems to be well tolerated in RTRs with glomerular filtration rate $>30 \mathrm{ml} / \mathrm{min} / 1.73 \mathrm{~m}^{2}$ [2]. Although controversial, a number of small studies have failed to prove the benefit of bisphosphonates on BMD in RTRs. Denosumab is an antibody against RANK-ligand, consequently decreasing bone resorption and increasing BMD. Because its elimination is independent of renal function it is a more attractive alternative compared to bisphosphonates. Although there are very few studies concerning the matter, denosumab was recently shown to effectively increase BMD in de novo RTRs [61].

\section{Orthopedic Management}

RTRs have a high potential for osteoarticular complications attributed to the chronic use of corticotherapy. Clinical practice patterns have changed over time as we've noticed a trend towards decreased steroid use. 
Naylor, et al. [62] estimated the 3, 5 and 10 year cumulative incidence of non-vertebral fractures in 4821 adult RTRs between 1994 and 2009. Only women recipients aged 50 years or older would be defined as a highrisk fracture group with $5.6 \%$ sustaining a hip fracture in the first 10 years after transplantation. Overall, recipients had a 3 times higher risk of fracture compared to the general population with no previous fracture. Even though RTRs may have a low absolute risk of fracture, they have a high relative risk compared to general healthy population.

The major orthopaedic sequela of chronic use of steroids is avascular necrosis (AVN) in major joints, particularly involving the femoral head [63]. In RTRs, bone ischemia often occurs within 12 weeks of transplantation [64]. A meta-analysis of forty-three studies demonstrated a sensitivity and specificity of MRI in early osteonecrosis of the femoral head of 93.0\% (95\% Cl 92.0-94.0\%) and 91.0\% (95\% Cl 89.0\%93.0\%), respectively [65]. Demirors, et al. [66] recommend early surgical intervention in RTRs as there is an extremely high failure rate (around 97\%) with conservative measures. Surgical options include core decompression, proximal femoral osteotomy or trapdoor grafting for early disease and hip resurfacing or total hip replacement (THR) in advanced stages [67].

With modern transplant surgery and clinical practice patterns RTRs are living longer and more active lives. They are increasingly likely to present to an arthroplasty surgeon for elective hip or knee replacement not only due to secondary AVN from chronic corticotherapy but also age-related development of osteoarthritis [68].

Cementing was considered the only viable method for fixation of hip implants in RTRs because of poor bone stock. Studies suggest that press-fit prostheses have similar survivorship in patients with good bone stock though long-term results for press-fit prostheses are still lacking [63]. The rate of bone loss is greatest in the first postoperative year and then progressively tapers off at around ten years after transplantation $[69,70]$.

An increased rate of complications after hip and knee arthroplasty in RTRs is well documented [71,72]. There are increased rates of periprosthetic infection and while pathogens are mainly caused by gram positive bacteria there are reports of opportunistic pathogens such as mycobacteria. Cultures should be sent for atypical infections, including anaerobes, mycobacteria and fungal [71] Tannenbaum, et al. [73] recommend that any new onset of renal pain in a RTR with a stable total hip replacement should be thoroughly investigated to exclude septic arthritis. Chronic immunosuppression has lasting consequences beyond infection risk including poor bone quality associated with periprosthetic fracture, instability and aseptic loosening of the components which contribute to an increased reoperation rate $[63,68]$.

Generally, immunosuppression regimens should be resumed pre and immediately postoperatively. Patients on sirolimus are an exception due to its association with an increased healing time and infection risk [74].

\section{Conclusion}

Bone disease in RTRs is complex and multifactorial. It is the result of CKD-MBD previous to transplant, residual renal function after transplant, immunosuppression and risk factors for bone disease common to the general population. The diagnosis of bone disease in RTRs is still difficult because of the lack of validated clinical scores and exams for this population. Recommended drugs for CKD patients (not RTRs) failed to prove significant beneficial impact. Recommended drugs for the general population are still controversial. RTRs have a low absolute risk of fracture but a high relative risk compared to the general population. These patients are increasingly likely to present to an arthroplasty surgeon for elective joint replacement not only due to secondary avascular necrosis but also age-related development of osteoarthritis. Periprosthetic infection, periprosthetic fracture and instability are increased in RTRs resulting in an increased reoperation rate.

\section{Disclosures}

Nothing to declare.

\section{References}

1. (2009) KDIGO Clinical Practice Guideline for the Diagnosis, Evaluation, Prevention, and Treatment of Chronic Kidney Disease-Mineral and Bone Disorder (CKD-MBD) Clinical practice, Kidney International 76.

2. KDIGO (2017) Clinical Practice Guideline Update for the Diagnosis, Evaluation, Prevention, and Treatment of Chronic Kidney Disease-Mineral and Bone Disorder (CKD-MBD) Kidney International 7: 1-59.

3. Slatolposky E, Gradowsky L, Kashemsa C (1966) Control of phosphate excretion in uremia. J Clin Invest 45: 672-677.

4. Shimada $T$, Hasegawa $H$, Yamazaki $Y$, Muto $T$, Hino R, et al. (2004) FGF-23 is a potent regulator of vitamin D metabolism and phosphate homeostasis. J Bone Miner Res 19: 429-435.

5. Gupta A, Winer K, Econs MJ (2004) FGF-23 is elevated by chronic hyperphosphatemia. J Clin Endocrinol Metab 89: 4489-4492.

6. Murer H, Hernando N, Forster I (2003) Regulation of $\mathrm{Na} /$ Pi transporter in the proximal tubule. Annu Rev Physiol 65: 531-542.

7. Kurosu H, Kuro-o M (2008) The Klotho gene family and the endocrine fibroblast growth factors. Curr Opin Nephrol Hypertens 17: 368-372.

8. Almaden Y, Canalejo A, Hernandez A, Ballesteros E, Garcia-Navarro S, et al. (1996) Direct effect of phosphorus on parathyroid hormone secretion from whole rat parathyroid glands in vitro. J Bone Miner Res 11: 970-976. 
9. Naveh-Many T, Rahamimov R, Livni N (1995) Parathyroid cell proliferation in normal and chronic renal failure rats: the effects of calcium, phosphate and vitamin D. J Clin Invest 96: 1786-1793.

10. Denda M, Finch J, Slatopolsky E (1996) Phosphorus accelerates the development of parathyroid hyperplasia and secondary hyperparathyroidism in rats with renal failure. Am J Kidney Dis 28: 596-602

11. Fu Q, Jilka RL, Manolagas SC, O'Brien CA (2002) Parathyroid hormone stimulates receptor activator of NFkappa B ligand and inhibits osteoprotegerin expression via protein kinase A activation of cAMP-response element-binding protein. J Biol Chem 277: 48868-48875.

12. Dusso AS, Brown AJ, Slatopolsky E (2005) Vitamin D. Am J Physiol Renal Physiol 289: F8-F28.

13. Lorenzo Sellares V, Torregrosa V (2008) Changes in mineral metabolism in stage 3,4 , and 5 chronic kidney disease (not on dialysis). Nefrologia Suppl 3: 67-78.

14. Laik V, Leaf EM, Hu JH, Hsueh-Ying Yang, Ngoc B Nguyen, et al. (2012) Sources of cells that contribute to atherosclerotic intimal calcification: An in vivo genetic fate mapping study. Cardiovascular Research 94: 545-554.

15. Kokubo T, Ishikawa N, Uchida H, Chasnoff SE, Xie X, et al. (2009) CKD accelerates development of neointimal hyperplasia in arteriovenous fistulas. J Am Soc Nephrol 20: 1236-1245.

16. Shanahan CM, Cary NRB, Salisbury JR, Proudfoot D, Weissberg PL, et al. (1999) Medial localization of mineralization-regulating proteins in association with Monckeberg's sclerosis: Evidence for smooth muscle cell-mediated vascular calcification. Circulation 100: 2168-2176.

17. Speer MY, Yang HY, Brabb T, Leaf E, Look A, et al. (2009) Smooth muscle cells give rise to osteochondrogenic precursors and chondrocytes in calcifying arteries. Circ Res 104: 733-741.

18. Zhu D, Mackenzie NCW, Shanahan CM, Shroff RC, Farquharson C, et al. (2015) BMP-9 regulates the osteoblastic differentiation and calcification of vascular smooth muscle cells through an ALK1 mediated pathway. J Cell Mol Med 19: $165-174$.

19. Agapova OA, Fang Y, Sugatani T, Seifert ME, Hruska KA (2016) Ligand trap for the activin type IIA receptor protects against vascular disease and renal fibrosis in mice with chronic kidney disease. Kidney Int 89: 1231-1243.

20. Fang Y, Ginsberg C, Sugatani T, Faugere MC, Malluche $\mathrm{H}$, et al. (2014) Early chronic kidney disease-mineral bone disorder stimulates vascular calcification. Kidney Int 85: 142-150.

21. Malluche HH, Monier-Faugere M-C, Herberth J (2010) Bone disease after renal transplantation. Nat Rev Nephrol 6: $32-40$.

22. Wolf M (2010) FGF23/iPTH and Phsophorus levels. J Am Soc Nephrol 21: 1427-1435.

23. Green J, Debby H, Lederer E, Levi M, Zajicek HK, et al. (2001) Evidence for a PTH-independent humoral mechanism in post-transplant hypophosphatemia and phosphaturia. Kidney Int 60: 1182-1196.

24. Trombetti A, Richert L, Hadaya K, Graf JD, Herrmann FR, et al. (2011) Early post-transplantation hypophosphatemia is associated with elevated FGF-23 levels. Eur J Endocrinol 164: 839-847.

25. Messa P, Cafforio C, Alfieri C (2010) Clinical impact of hy- percalcemia after kidney transplant. G Ital Nefrol 27: 47-55.

26. Lou I, Foley D, Odorico SK, Leverson G, Schneider DF, et al. (2015) How well does renal transplantation cure hyperparathyroidism? Ann Surg 262: 653-659.

27. Maria Concetta Gioviale, Maurizio Bellavia, Giuseppe Damiano, Attilio Ignazio Lo Monte (2012) Post-transplantation tertiary hyperparathyroidism. Ann Transplant 17: 111-119.

28. Akaberi S, Lindergård B, Simonsen O, Nyberg G (2006) Impact of parathyroid hormone on bone density in long-term renal transplant patients with good graft function. Transplantation 82: 749-752.

29. Antoine Bouquegneau, Syrazah Salam, Pierre Delanaye, Richard Eastell, ArifKhwaja (2016) Bone disease after kidney transplantation. Clin J Am Soc Nephrol 11: 1282-1296.

30. Perrin P, Caillard S, Javier RM, Braun L, Heibel F, et al. (2013) Persistent hyperparathyroidism is a major risk factor for fractures in the five years after kidney transplantation. Am J Transplant 13: 2653-2663.

31. Kanaan N, Claes K, Devogelaer JP, Vanderschueren D, Depresseux G, et al. (2010) Fibroblast growth factor-23 and parathyroid hormone are associated with post transplant bone mineral density loss. Clin J Am Soc Nephrol 5: 1887-1892.

32. Monier-Faugere MC, Mawad H, Qi Q, Friedler RM, Malluche $\mathrm{HH}(2000)$ High prevalence of low bone turnover and occurrence of osteomalacia after kidney transplantation. $J$ Am Soc Nephrol 11: 1093-1099.

33. Julian BA, Laskow DA, Dubovsky J, Dubovsky EV, Curtis JJ, et al. (1991) Rapid loss of vertebral mineral density after renal transplantation. N Engl J Med 325: 544-550.

34. Van Staa TP, Leufkens HG, Abenhaim L, Zhang B, Cooper C (2000) Use of oral corticosteroids and risk of fractures. J Bone Miner Res 15: 993-1000.

35. Grotz WH, Breitenfeldt MK, Braune SW, Allmann $\mathrm{KH}$, Krause TM, et al. (2001) Calcineurin-inhibitor induced pain syndrome (CIPS): A severe disabling complication after organ transplantation. Transpl Int 14: 16-23.

36. Sun L, Blair HC, Peng Y, Zaidi N, Adebanjo OA, et al. (2005) Calcineurin regulates bone formation by the osteoblast. Proc Natl Acad Sci U S A 102 : 17130-17135.

37. Fukunaga J, Yamaai T, Yamachika E, Ishiwari Y, Tsujigiwa $\mathrm{H}$, et al. (2004) Expression of osteoclast differentiation factor and osteoclastogenesis inhibitory factor in rat osteoporosis induced by immunosuppressant FK506. Bone 34: 425-431.

38. Singha UK, Jiang Y, Yu S, Luo M, Lu Y, et al. (2008) Rapamycin inhibits osteoblast proliferation and differentiation in MC3T3-E1 cells and primary mouse bone marrow stromal cells. J Cell Biochem 103: 434-446.

39. Xian L, Wu X, Pang L, Lou M, Rosen CJ, et al. (2012) Matrix IGF-1 maintains bone mass by activation of mTOR in mesenchymal stem cells. Nat Med 18: 1095-1101.

40. NIH Consensus Development Panel on Osteoporosis Prevention, Diagnosis, and Therapy (2001) Osteoporosis prevention, diagnosis, and therapy. JAMA 285: 785-795.

41. Kanis JA, Melton LJ 3rd, Christiansen C, Johnston CC, Khaltaev N (1994) The diagnosis of osteoporosis. Journal of Bone and Mineral Research 9: 1137-1141.

42. Assessment of fracture risk and its application to screening for postmenopausal osteoporosis (1994) Report of a WHO study group. Geneva, World Health Organization. 
43. Kanis JA, Oden A, Johnell O, Johansson H, De Laet $C$ et al. (2007) The use of clinical risk factors enhances the performance of BMD in the prediction of hip and osteoporotic fractures in men and women. Osteoporos Int 18: 10331046.

44. D'Amelio P, Isaia GC (2015) Male osteoporosis in the elderly. Int J Endocrinol 2015: 907689.

45. De Laet C, Kanis JA, Oden A, Johanson H, Johnell O, et al. (2005) Body mass index as a predictor of fracture risk: $A$ meta-analysis. Osteoporos Int 16: 1330-1038.

46. Kanis JA, Johnell O, De Laet C, Johansson H, Oden A, et al. (2004) A meta-analysis of previous fracture and subsequent fracture risk. Bone 35: 375-382.

47. Fox KM, Cummings SR, Powell-Threets K, Stone K (1998) Family history and risk of osteoporotic fracture. Study of osteoporotic fractures research group. Osteoporos Int 8: 557-562.

48. Kanis JA, Johnell O, Oden A, Johansson H, De Laet C, et al. (2005) Smoking and fracture risk: A meta-analysis. Osteoporos Int 16: 155-162.

49. Kanis JA, Stevenson M, McCloskey EV, Davis S, LloydJones M (2007) Glucocorticoid-induced osteoporosis: A systematic review and cost-utility analysis. Health Technol Assess 11: 1-231.

50. Kanis JA, Johansson $H$, Johnell O, Oden A, De Laet C, et al. (2005) Alcohol intake as a risk factor for fracture. Osteoporos Int 16: 737-742.

51. Sarkis KS, Salvador MB, Pinheiro MM, Silva RG, Zerbin CA, et al. (2009) Association between osteoporosis and rheumatoid arthritis in women: A cross-sectional study. Sao Paulo Med J 127: 216-222.

52. Guidelines for preclinical evaluation and clinical trials in osteoporosis (1998) Geneva, World Health Organization.

53. Committee for Proprietary Medicinal Products (2001) Note for guidance on postmenopausal osteoporosis in women. European Agency for the Evaluation of Medicinal Products, London.

54. Ketteler M, Block GA, Evenepoel P, Fukagawa M, Herzog $\mathrm{CA}$, et al. (2017) Executive summary of the $2017 \mathrm{KDI}$ GO chronic kidney disease-mineral and bone disorder (CKDMBD) guideline update: What's changed and why it matters. Kidney Int 92: 26-36.

55. Kansal S, Fried L (2010) Bone disease in elderly individuals with CKD. Adv Chronic Kidney Dis 17: E41-E51.

56. Diamond T, Elder GJ (2017) Is there a practical role for bone biopsy in chronic kidney disease? Nephrology (Carlton) 22: 22-26.

57. Carvalho C, Alves CM, Frazão JM (2016) The role of bone biopsy for the diagnosis of renal osteodystrophy: A short overview and future perspectives. J Nephrol 29: 617-26.

58. Evenepoel P, Cooper K, Holdaas H, Messa P, Mourad G, et al. (2014) A randomized study evaluating cinacalcet to treat hypercalcemia in renal transplant recipients with persistent hyperparathyroidism. Am J Transplant 14: 2545-2555.

59. Peacock M, Bolognese MA, Borofsky M, Scumpia S, Sterling LR, et al. (2009) Cinacalcet treatment of primary hyperparathyroidism: Biochemical and bone densitometric outcomes in a five-year study. J Clin Endocrinol Metab 94: 4860-4867.

60. Russell RG, Muhlbauer RC, Bisaz S, Williams DA, Fleisch $H(1970)$ The influence of pyrophosphate, condensed phos- phates, phosphonates and other phosphate compounds on the dissolution of hydroxyapatite in vitro and on bone resorption induced by parathyroid hormone in tissue culture and in thyroparathyroidectomised rats. Calcif Tissue Res 6: $183-196$

61. Bonani M, Frey D, Brockmann J, Fehr T, Mueller TF, et al. (2016) Effect of twice-yearly denosumab on prevention of bone mineral density loss in de novo kidney transplant recipients: A randomized controlled trial. Am J Transplant 16: 1882-1891.

62. Naylor KL, Jamal SA, Zou G, McArthur E, Lam NN, et al. (2016) 100: 167-175 Fracture incidence in adult kidney transplant recipients. Transplantation 100: 165-175.

63. Nowicky $P$, Chaudhary $H$ (2007) Total hip replacement in renal transplant patients. J Bone Joint Surg 89: 1561-1566.

64. Asano T, Takahashi K, Fujioka M, Inoue S, Satomi Y, et al. (2003) Genetic analysis of steroid-induced osteonecrosis of the femoral head. J Orthop Sci 8: 329-333.

65. Zhang YZ, Cao XY, Li XC, Chen J, Zhao YY, et al. (2018) Accuracy of MRI diagnosis of early osteonecrosis of the femoral head: a meta-analysis and systematic review. J Orthop Surg Res 13: 167.

66. Demirors H, Kaya A, Akpinar S, Tuncay C, Tandogan RN (2002) Effect of long-term steroiduse on prognosis for patients with surgically treated avascular necrosis of the hip. Transplant Proc 34: 2114-2118.

67. Beaulé PE, Amstutz HC (2004) Management of Ficat Stage III and IV osteonecrosis of the hip. J Am Acad Orthop Surg 12: $96-105$.

68. Brian T Nickel, Cameron K Ledford, Tyler Steven Watters, Samuel S Wellman, Michael P Bolognesi (2015) Arthroplasty in organ transplant patients. Arthroplasty Today 1: 41-44.

69. Carlini RG, Rojas E, Weisinger JR, Lopez M, Martinis R, et al. (2000) Bone disease in patients with long-term renal transplantation and normal renal function. Am J Kidney Dis 36: 160-166.

70. Kodras K, Haas M (2006) Effect of kidney transplantation on bone. European Journal of Clinical Investigation 36: 6375 .

71. Vergidis P, Lesnick TG, Kremers WK (2012) Prosthetic joint infection in solid organ transplant recipients: A retrospective case-control study. Transpl Infect Dis 14: 380-386.

72. Cavanaugh P, Chen A, Rasouli M, Post ZD, Orozco FR, et al. (2015) Total joint arthroplasty in transplant recipients: In-hospital adverse outcomes. J Arthroplasty 30: 840-845.

73. Tannenbaum DA, Matthews LS, Grady-Benson JC (1997) Infection around joint replacements in patients who have a renal or liver transplantation. J Bone Joint Surg 79: 36-43.

74. Fortun J, Martin-Davila P, Pascual J, Cervera C, Moreno A, et al. (2010) Immunosuppressive therapy and infection after kidney transplantation. Transpl Infect Dis 12: 397-405. 